\title{
Intervenciones asistidas con perros en alumnado con Trastorno del Espectro Autista. Una revisión de la literatura
}

María Poveda Gómez - Universitat de València

Diana Marín Suelves - Universitat de València

Sandra Navarro Sánchez - Universitat de València

$0000-0002-9702-8450$

(D) $0000-0002-5346-8665$

(D) 0000-0003-2585-7804

Recepción: 16.09.2021 | Aceptado: 19.10.2021

Correspondencia a través de ORCID: Diana Marín Suelves

iD 0000-0002-5346-8665

Citar: Poveda Gómez, M, Marín Suelves, D y Navarro Sánchez, S (2021). Intervenciones asistidas con perros en alumnado con Trastorno del Espectro Autista. Una revisión de la literatura. REIDOCREA, 10(38), $1-12$.

Área o categoría del conocimiento: Educación

Resumen: El presente trabajo constituye una revisión bibliográfica sobre la intervención asistida con perros en niños con trastorno del espectro autista en edad escolar. Los objetivos de la investigación son conocer las metas que persiguen las intervenciones asistidas con perros en niños con trastorno del espectro autista, los resultados obtenidos y cómo y dónde se realizan. Para ello se ha realizado una revisión de la literatura en base a los artículos de investigación publicados a partir del año 2000 en las bases de datos Web of Science (Wos), Scopus, PubMed y CINAHL. Los resultados obtenidos han confirmado los beneficios de las intervenciones asistidas con perros en niños con trastorno del espectro autista, mejorando las habilidades e interacciones sociales, que conllevan un aumento de la comunicación, minimizan comportamientos sociales negativos y aumentan los positivos, generan confianza y autoestima en los niños con trastorno del espectro autista y mejoran las relaciones y tiempo de recreación familiar. Finalmente, este trabajo expone las limitaciones observadas de los estudios revisados y propone una serie de cuestiones para futuras investigaciones o líneas de trabajo que sería conveniente explorar.

Palabra clave: Trastorno del espectro autista

\section{Dog-assisted interventions in students with Autism Spectrum Disorder. A review of the literature}

\begin{abstract}
The present work constitutes a bibliographic review on dog-assisted intervention in school-age children with autism spectrum disorder. The objectives of the research are to know the goals of dog-assisted interventions in children with autism spectrum disorder, the results obtained and how and where they are carried out. To this end, a literature review has been carried out based on research articles published since 2000 in the Web of Science (Wos), Scopus, PubMed and CINAHL databases. The results obtained have confirmed the benefits of dog-assisted interventions in children with autism spectrum disorder, improving skills and social interactions, leading to increased communication, minimizing negative social behaviors and increasing positive ones, generating confidence and self-esteem in children with autism spectrum disorder improve relationships and family recreation time. Finally, this work exposes the observed limitations of the reviewed studies and proposes a series of questions for future research or lines of work that it would be convenient to explore.
\end{abstract}

Keyword: Autism Spectrum Disorder

\section{Introducción}

En España la prevalencia del Trastorno de Espectro Autista (TEA) se estima en un $0.61 \%$ de los niños en edad escolar (Fortea et al., 2013). Sin embargo, se debe comprender que la prevalencia de un mismo diagnóstico cambia o puede ser muy diferente dependiendo de la coyuntura y el lugar en el que se lleve a término. Nos obstante, existen dos factores que destacan notablemente en la prevalencia del TEA, tales como el género y la presencia de comorbilidad. EI TEA es cuatro veces más habitual en el género masculino que en el femenino. Y, por otro lado, la prevalencia del TEA se ve alterada por el diagnóstico comórbido de índole variada. A lo largo de las últimas décadas ha habido una tendencia creciente en la incidencia del TEA que podría deberse al aumento de la prevalencia a causa de factores metodológicos a la hora de 
llevar a cabo el diagnóstico o podría deberse a un aumento en la incidencia del trastorno de espectro autista (Fombonne, 2009).

Por lo que respecta a la etiología del TEA, debe tenerse en cuenta que actualmente no se conocen las causas que explican su aparición y no es posible determinar una única causa que tenga relación directa con su presencia. Sin embargo, diferentes estudios sí reconocen factores genéticos, epigenéticos y ambientales. En primer lugar, por lo que respecta a los factores genéticos, se advierte que la probabilidad de que un niño presente TEA oscila entre un $2 \%$ y un $8 \%$ superior en los casos de niños con hermanos con diagnóstico TEA (Chaste y Leboyer, 2012). Asimismo, estudios entre gemelos monocigóticos, es decir que comparten el $100 \%$ de los genes, han revelado que cuando un hermano monocigótico tiene TEA, es más probable que su hermano gemelo también lo tenga. Por el contrario, esta coincidencia no ocurre con tanta asiduidad en hermanos gemelos dicigóticos. Respecto a la relación con la epigenética, referida a los procesos que modulan la expresión de los genes, que son la causa de numerosos síndromes genéticos que se relacionan con el TEA, coocurren con la sintomatología TEA. Finalmente, existen factores ambientales, como los prenatales, perinatales y posnatales que manifiestan un aumento del riesgo o una mayor probabilidad de presentar TEA (Tárraga et al., 2018).

Con la clasificación del TEA del DSM-5 (APA, 2013), se origina una nueva conceptualización con perspectiva dimensional del autismo, entendido como un espectro de trastornos de severidad diversa (Bonilla y Chaskel, 2016). En la Tabla 1 se muestra el resumen de los niveles de gravedad del TEA.

Tabla 1. Niveles de gravedad del trastorno del espectro autista (Bonilla y Chaskel, 2016).

\begin{tabular}{|l|l|l|}
\hline $\begin{array}{l}\text { Categoría dimensional del TEA en } \\
\text { el DSM-5 }\end{array}$ & Comunicación social & Comportamientos restringidos y repetitivos \\
\hline $\begin{array}{l}\text { Grado 3: "Necesita ayuda muy } \\
\text { notable" }\end{array}$ & $\begin{array}{l}\text { Déficit grave en la comunicación } \\
\text { social verbal y no verbal que causan } \\
\text { problemas en la interacción social. }\end{array}$ & $\begin{array}{l}\text { Extrema inflexibilidad y dificultades para hacer } \\
\text { frente a cambios. Ansiedad intensa al cambiar } \\
\text { de foco de atención. }\end{array}$ \\
\hline Grado 2: "Necesita ayuda notable" & $\begin{array}{l}\text { Déficit notable en la comunicación } \\
\text { social verbal y no verbal. }\end{array}$ & $\begin{array}{l}\text { Inflexibilidad del comportamiento y dificultades } \\
\text { para cambiar el foco de interés que crean } \\
\text { ansiedad o dificultad. }\end{array}$ \\
\hline Grado 1: "Necesita ayuda" & $\begin{array}{l}\text { Alteraciones significativas en el área } \\
\text { de la comunicación social. }\end{array}$ & $\begin{array}{l}\text { La inflexibilidad del comportamiento causa } \\
\text { interferencia significativa en uno o más } \\
\text { contextos y dificulta la autonomía. }\end{array}$ \\
\hline
\end{tabular}

Asimismo, las necesidades educativas del alumnado con TEA tienen relación directa con los criterios diagnósticos descritos según el DSM-5 y, por consiguiente, estas necesidades educativas se pueden aunar en dos grupos, tales como:

- Necesidades educativas relacionadas con la comunicación e interacción social. Es recomendable una educación emocional donde se aprenda a conocer, expresar e interpretar las emociones propias y las ajenas, además de aprender ciertas habilidades sociales que les faciliten las relaciones sociales afectivas.

- Necesidades educativas en relación con los patrones de comportamiento, intereses y actividades restringidas y estereotipadas. Es conveniente realizar actividades estructuradas, incluyendo cambios en la rutina para evitar patrones de comportamiento y crear nuevos focos de intereses.

La terapia asistida con animales es una de las intervenciones que se emplean en la actualidad para la superación de las dificultades asociadas al TEA. 


\section{Terapias asistidas con animales}

En la actualidad, las intervenciones asistidas con animales (IAA) son pensadas como un programa de intervención estructurado, con objetivos terapéuticos, sociales, educativos o preventivos, diseñados para mejorar la calidad de vida de los sujetes con los que se interviene mediante animales previamente seleccionados, bajo las directrices y supervisión de los profesionales que participan en cada programa (International Association of Human-Animal Interaction Organizations, 2014).

Siguiendo a Ristol y Domènec (2013), dentro de las IAA se contemplan diversas formas de interacción entre los animales y las personas como son: las Actividades Asistidas con Animales (AAA), la Terapia Asistida con Animales (TAA), la Educación Asistida con Animales (EAA), Valoración Asistida con Animales (VAA) y Programas de Animal Residente (PAR). Por otro lado, se encuentran diferentes agentes que participan en las IAA como el profesional de la intervención, pudiendo ser especialistas de la salud, de veterinaria o de educación, previamente formados con los conocimientos necesarios para la aplicación de estas intervenciones, además de la unidad de intervención, constituida por el técnico en intervenciones asistidas con animales y un animal seleccionado y adiestrado para dichas intervenciones.

Tal y como afirma la Fundación Affinity (2019), las terapias asistidas con animales son muy beneficiosas para las personas con algún tipo de diagnóstico, debido a que mejoran las relaciones sociales con el medio debido la atención que estas terapias asistidas con animales despiertan en los niños con TEA. El vínculo que se crea entre el animal y el individuo es el punto de inflexión para comenzar a trabajar la comunicación, las relaciones y la apertura emocional, fortaleciendo así su autoestima, entrenando la motricidad fina y gruesa, propiciando escenarios de socialización y desarrollando las habilidades comunicativas, ya que los animales ofrecen una estimulación permanente para los niños con TEA.

Sin embargo, en esta investigación se centra el objeto de estudio en las terapias asistidas con perros, dado que como sugiere Signes (2009), los perros son animales idóneos para desempeñar cualquier actividad o intervención asistida con animales, dado que ofrecen muchas ventajas frente a otros animales como son:

- Estímulo multisensorial para los niños con TEA.

- Los perros son un facilitador social.

- El perro puede ser utilizado por los niños con TEA como un objeto transicional.

- Los perros son más accesibles que otros animales.

- Mediante este tipo de terapias asistidas con perros para niños con TEA se pueden trabajar 4 áreas principalmente, clasificadas por Jara y Chacón (2017) en: área física, área cognitiva, área emocional, área relacional.

\section{Método}

El presente trabajo se ha centrado en la realización de una revisión bibliográfica durante el mes de abril de 2021, mediante la búsqueda en diferentes bases de datos de artículo científicos. Las bases de datos empleadas han sido Web of Science (Wos), PubMed, Scopus y CINAHL, donde se emplearon los descriptores "Canine-assisted", "Education", "Autistic disorder", "dog", "Autism spectrum disorder", "education" y "Children", combinados con los operadores booleano "AND" y "OR". 
Del mismo modo, dicha revisión bibliográfica está sustentada en las directrices y la lista de verificación de la Declaración PRISMA (Urrútia y Bonfill, 2010), siendo los criterios de inclusión y exclusión utilizados los mostrados en la Tabla 2.

Tabla 2. Criterios de inclusión y exclusión

\begin{tabular}{|l|l|}
\hline & Criterios \\
\hline Inclusión & - Artículos de investigación en acceso abierto. \\
& - Artículos publicados entre 2000 y el primer trimestre de 2021. \\
& - Artículos donde la población de estudio fuesen niños en edad escolar con TEA o las personas \\
encargadas de la intervención. & - Artículos en español e inglés. \\
\hline Exclusión & - Tipología de documentos diferente a artículos. \\
& - Artículos científicos alejados del objeto de estudio. \\
& - Revisiones de la literatura. \\
& - Artículos basados en intervenciones realizadas con otros animales. \\
& - Artículos donde la población de estudio fueran personas adultas con TEA. \\
& - Artículos duplicados. \\
\hline
\end{tabular}

En la Figura 1 se muestra el proceso seguido para la selección de los 19 artículos que componen este estudio.

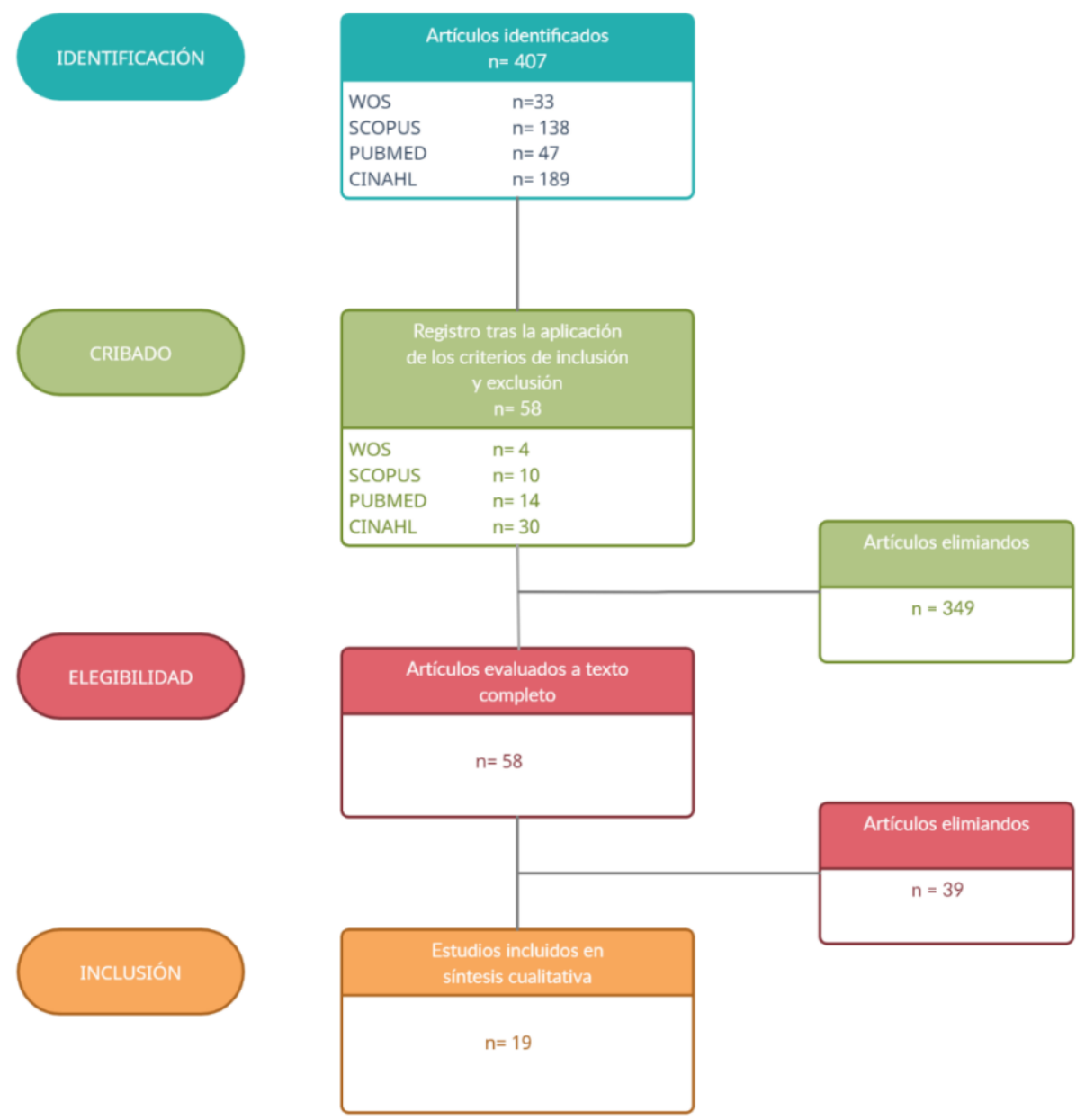

Figura 1. Diagrama de flujo sobre las fases de la revisión bibliográfica. 
Resultados

Las principales características de los 19 artículos seleccionados se resumen en la Tabla 3.

Tabla 3: Artículos científicos sobre intervenciones asistidas con perros en niños con TEA.

\begin{tabular}{ll}
\hline Autores & Objetivos \\
\hline Hill, Ziviani y & Buscar una \\
Driscoll & comprensión de los \\
$(2020$, & posibles beneficios y \\
Australia) & desafíos de la \\
& práctica de las \\
& intervenciones \\
& asistidas con perros.
\end{tabular}

$\begin{array}{ll}\text { Muestra } & \text { Intervención } \\ \mathrm{N}=6 \text { terapeutas } & \text { Evaluación de las } \\ \text { ocupacionales } & \text { respuestas obtenidas en } \\ \text { de niños con } & \text { las entrevistas de las } \\ \text { TEA. } & \text { terapeutas ocupacionales } \\ & \text { que realizan } \\ & \text { intervenciones asistidas } \\ & \text { con perros en niños con } \\ & \text { TEA. }\end{array}$

Resultados

Introducir el perro de terapia

acelera la motivación de los niños con TEA en las sesiones, facilitando de este modo el desarrollo de las sesiones, la realización de las tareas de forma autónoma y aumentando la confianza. Por el contrario, es un desafío mantener el enfoque de los objetivos al incluir el perro de terapia.

\begin{tabular}{ll}
\hline Hill, Ziviani, & Exploración del \\
Discroll, & impacto de la terapia \\
Teoh, Chua y & ocupacional asistida \\
Cawdell & con perros en los \\
(2020, & comportamientos en \\
Australia) & la tarea y el logro de \\
& metas de los niños \\
& con TEA.
\end{tabular}

$\begin{array}{ll}\mathrm{N}=22 \text { niños con } & \text { Evaluación de las } \\ \mathrm{TEA} & \text { actividades de terapia } \\ \mathrm{E}=4-6 \text { años. } & \text { ocupacional, actitudes y } \\ & \text { comportamiento hacia los } \\ & \text { perros. }\end{array}$

El grupo experimental que trabajó con el perro de terapia mejoró el comportamiento en las tareas y el rendimiento. No obstante, estos resultados no destacan del grupo control que trabajó sin perro. Hay que añadir que los esfuerzos por parte de los terapeutas del grupo control para mantener la atención de los niños cuando el perro no estuvo presente fueron más elevados que el del grupo experimental. Por otro lado, algunos comportamientos desafiantes como la hiperactividad, la agresión y las dificultades atencionales de los niños con TEA pueden perjudicar en ocasiones al perro de terapia.

\begin{tabular}{lll}
\hline Carlisle et al. & Explorar la propiedad & $\mathrm{N}=764$ padres \\
(2020, EE. & de los animales de & de niños con \\
UU.) & compañía en las & TEA \\
& familias de niños con & $\mathrm{E}=3-18$ años. \\
& TEA.
\end{tabular}

Examinar los vínculos de la familia con el perro de compañía, la percepción de los padres sobre los beneficios familiares, las cargas de poseer un perro, el vínculo que se crea entre el perro y el niño e identificar los riesgos de poseer un perro como animal de compañía para un niño con TEA.

\begin{tabular}{|c|c|c|c|}
\hline $\begin{array}{l}\text { Morales et al. } \\
\text { (2020, } \\
\text { España) }\end{array}$ & $\begin{array}{l}\text { Trabajar con niños } \\
\text { con TEA de forma } \\
\text { multidisciplinar } \\
\text { mediante el uso de } \\
\text { las teranias asistidas }\end{array}$ & $\begin{array}{l}\mathrm{N}=16 \text { niños con } \\
\text { TEA. }\end{array}$ & $\begin{array}{l}\text { Evaluación de } \\
\text { comportamientos, } \\
\text { emociones y análisis del } \\
\text { lenguaje. }\end{array}$ \\
\hline
\end{tabular}
con perros.
Beneficios y aumento de las interacciones sociales positivas de los niños con TEA al poseer una mascota, las responsabilidades de tener una mascota no interfieren en el estrés de la familia y creación de un vínculo tanto de la familia como de niño con TEA con el animal.

Mejoras en el lenguaje gestual,
iniciación de conversaciones y
mantenimiento de estas, mejora
de la ecolalia, mejora de la
emisión de sonidos vocales y
palabras o frases estereotipadas
y aumento del comportamiento
social positivo.
Las relaciones de imitación
mejoran y ocurren con más
frecuencia frente al perro igual
que aparecen menos, emociones
negativas para los niños con
TEA.




\begin{tabular}{|c|c|c|}
\hline $\begin{array}{l}\text { Protopopova } \\
\text { et al. (2020, } \\
\text { EE. UU.) }\end{array}$ & $\begin{array}{l}\text { Comparación del } \\
\text { acceso contingente y } \\
\text { no contingente de } \\
\text { perros de terapia a } \\
\text { los niños con TEA } \\
\text { durante tareas } \\
\text { educativas. }\end{array}$ & $\begin{array}{l}\mathrm{N}=5 \text { niños con } \\
\text { TEA } \\
\mathrm{S}=4 \bigcirc 1 \text { } \\
\mathrm{E}=7-11 \text { años }\end{array}$ \\
\hline
\end{tabular}

Evaluación de las tareas, entrenamientos de interacción con el perro, entrenamiento de tokens, evaluación de preferencias y muestra de saliva para calcular niveles de cortisol.
Los niños con TEA respondieron correctamente con más frecuencia y tuvieron un comportamiento más adecuado hacia la tarea cuando se presentó al perro de manera contingente que de manera no contingente. No obstante, el comportamiento social positivo ocurría con más frecuencia cuando el perro era mostrado de manera no contingente.

\begin{tabular}{|c|c|c|c|}
\hline $\begin{array}{l}\text { Griffien et al. } \\
\text { (2019, Países } \\
\text { Bajos) }\end{array}$ & $\begin{array}{l}\text { Comparación de los } \\
\text { patrones de } \\
\text { movimientos y los } \\
\text { perros de terapia } \\
\text { para comprobar los } \\
\text { cambios en }\end{array}$ & $\begin{array}{l}\mathrm{N}=10 \text { niños con } \\
\text { TEA y Síndrome } \\
\text { de Down } \\
\mathrm{S}=80^{\lambda} 2 \text { 2 } \\
\mathrm{E}=\text { media de } 12 \\
\text { años }\end{array}$ & $\begin{array}{l}\text { Evaluación del movimiento } \\
\text { del niño y el } \\
\text { comportamiento } \\
\text { demostrado hacia el perro. }\end{array}$ \\
\hline
\end{tabular}
Mayor proporción de sincronía entre los niños y los perros y disminución de los comportamientos problemáticos.

problemas

emocionales y

conductuales.

\begin{tabular}{ll}
\hline Uccheddu et & Comprobación de la \\
al. (2019, & hipótesis de que leer \\
Italia y & frente a un perro \\
Bélgica) & mejora la motivación \\
& hacia la lectura de los \\
& niños con TEA.
\end{tabular}

$\mathrm{N}=7$ niños con
TEA
$\mathrm{S}=7 \lesssim 2$ 2 2
$\mathrm{E}=6-11$ años.

Se evaluó la precisión, No hubo diferencias significantes velocidad, comprensión, entre el grupo experimental que reconocimiento, fluidez, leía frente a un perro de lectura y fonémico, segmentación y el grupo de control que leía eliminación de letras en la enfrente del maestro. Un dato lectura. que resaltar es que los niños del grupo experimental aumentaron las ganas de leer en casa y mirar libros de manera autónoma, notándose más motivación en la lectura dado que el perro creaba confianza en los niños con TEA.

\begin{tabular}{|c|c|c|c|c|}
\hline $\begin{array}{l}\text { Barber y } \\
\text { Proops (2019, } \\
\text { Reino Unido) }\end{array}$ & $\begin{array}{l}\text { Estudiar el efecto de } \\
\text { la lectura en voz alta } \\
\text { de los niños enfrente } \\
\text { de los perros de } \\
\text { terapia y de las } \\
\text { maestras. }\end{array}$ & $\begin{array}{l}\mathrm{N}=20 \text { niños con } \\
\text { TEA, Síndrome } \\
\text { de Down, } \\
\text { dislexia, } \\
\text { ansiedad y } \\
\text { fibrosis quística } \\
\mathrm{E}=6-11 \text { años }\end{array}$ & $\begin{array}{l}\text { Evaluación de la lectura } \\
\text { enfrente del maestro y del } \\
\text { perro de terapia. }\end{array}$ & $\begin{array}{l}\text { Los estudiantes leían más fluido, } \\
\text { habian mejorado el ritmo de } \\
\text { lectura y habían mejorado el } \\
\text { comportamiento de lectura } \\
\text { respecto a la postura y confianza } \\
\text { enfrente del perro. }\end{array}$ \\
\hline $\begin{array}{l}\text { Ávila et al. } \\
(2019, \\
\text { España) }\end{array}$ & $\begin{array}{l}\text { Exploración de la } \\
\text { viabilidad de realizar } \\
\text { intervenciones } \\
\text { tempranas con perros } \\
\text { de terapia para la } \\
\text { mejora de las } \\
\text { habilidades } \\
\text { comunicativas y la } \\
\text { interacción social. }\end{array}$ & $\begin{array}{l}\mathrm{N}=19 \text { niños con } \\
T E A \\
\mathrm{E}=\text { edad media } \\
\text { de } 3 \text { años. }\end{array}$ & $\begin{array}{l}\text { Evaluación de las } \\
\text { habilidades comunicativas } \\
\text { e interacción social } \\
\text { durante las intervenciones } \\
\text { y el comportamiento del } \\
\text { niño con TEA respecto a } \\
\text { las interacciones con el } \\
\text { perro y los terapeutas. }\end{array}$ & $\begin{array}{l}\text { La aplicación temprana de una } \\
\text { intervención basada en perros de } \\
\text { terapia es factible en niños con } \\
\text { TEA, ya que mejora las } \\
\text { habilidades de comunicación e } \\
\text { interacción social, facilitando la } \\
\text { participación social. }\end{array}$ \\
\hline $\begin{array}{l}\text { Germone et } \\
\text { al. (2019, EE. } \\
\text { UU.) }\end{array}$ & $\begin{array}{l}\text { Estudiar la mejora de } \\
\text { los comportamientos } \\
\text { sociales mediante las } \\
\text { actividades asistidas } \\
\text { por perros en niños } \\
\text { con TEA. }\end{array}$ & $\begin{array}{l}\mathrm{N}=47 \text { niños con } \\
\text { TEA } \\
\mathrm{E}=6-8 \text { años. }\end{array}$ & $\begin{array}{l}\text { Evaluación de los } \\
\text { comportamientos sociales } \\
\text { y comunicativos. }\end{array}$ & $\begin{array}{l}\text { Los niños con TEA mostraron } \\
\text { comportamientos más sociales y } \\
\text { comunicativos con uso de } \\
\text { gestos, sostenimiento de la } \\
\text { mirada, expresiones faciales } \\
\text { positivas y vocalizaciones } \\
\text { positivas con los perros de } \\
\text { terapia. }\end{array}$ \\
\hline $\begin{array}{l}\text { Guérin et al. } \\
(2018, \text { EE. } \\
\text { UU.) }\end{array}$ & $\begin{array}{l}\text { Fiabilidad y validez } \\
\text { de la herramienta } \\
\text { OHAIRE durante la } \\
\text { intervención asistida } \\
\text { con animales en } \\
\text { niños. }\end{array}$ & $\begin{array}{l}\mathrm{N}=71 \text { niños con } \\
\text { TEA y } 125 \\
\text { niños con TDAH } \\
\text { y niños } \\
\text { neurotípicos. }\end{array}$ & $\begin{array}{l}\text { Evaluación de las } \\
\text { interacciones sociales, la } \\
\text { comunicación social, los } \\
\text { comportamientos e } \\
\text { interacciones con } \\
\text { animales y objetos de } \\
\text { control a través del } \\
\text { análisis de vídeos. }\end{array}$ & $\begin{array}{l}\text { El OHAIRE demostró una buena } \\
\text { fiabilidad, demostrando también } \\
\text { el grado de bienestar animal en } \\
\text { las intervenciones asistidas con } \\
\text { perros. }\end{array}$ \\
\hline $\begin{array}{l}\text { Hall, Wright y } \\
\text { Mills (2017, } \\
\text { EE. UU.) }\end{array}$ & $\begin{array}{l}\text { Percepción de las } \\
\text { familias sobre la } \\
\text { calidad de vida de los }\end{array}$ & $\begin{array}{l}\mathrm{N}=36 \text { madres } \\
\text { de niños con } \\
\text { TEA y con }\end{array}$ & $\begin{array}{l}\text { Observación de los } \\
\text { comportamientos de } \\
\text { ansiedad de los perros. }\end{array}$ & $\begin{array}{l}\text { Los niños aportan la rutina } \\
\text { necesaria a los perros, } \\
\text { actividades de alta energía y se }\end{array}$ \\
\hline
\end{tabular}




\begin{tabular}{|c|c|c|c|c|}
\hline & $\begin{array}{l}\text { perros que viven en } \\
\text { el hogar de niños con } \\
\text { TEA. }\end{array}$ & $\begin{array}{l}\text { desarrollo } \\
\text { neurotípico } \\
E=4-10 \text { años. }\end{array}$ & & $\begin{array}{l}\text { establece un fuerte vínculo } \\
\text { humano-animal. No obstante, los } \\
\text { niños con TEA mostraron una } \\
\text { serie de factores negativos para } \\
\text { la calidad de vida de los perros } \\
\text { como es la invasión de su } \\
\text { espacio persona o agresión } \\
\text { física infantil. }\end{array}$ \\
\hline $\begin{array}{l}\text { Hall, Wright y } \\
\text { Mills (2016, } \\
\text { Reino Unido) }\end{array}$ & $\begin{array}{l}\text { Desarrollo de una } \\
\text { escala basada en el } \\
\text { impacto percibido por } \\
\text { los padres de niños } \\
\text { con TEA sobre la } \\
\text { adquisición de un } \\
\text { perro de asistencia o } \\
\text { servicio. }\end{array}$ & $\begin{array}{l}\mathrm{N}=197 \text { padres } \\
\text { de niños con } \\
\text { TEA } \\
\mathrm{E}=2-11 \text { años. }\end{array}$ & $\begin{array}{l}\text { Evaluación de la influencia } \\
\text { de los perros de compañía } \\
\text { en el desarrollo de las } \\
\text { familias de niños con TEA. }\end{array}$ & $\begin{array}{l}\text { Mejoras generales en la } \\
\text { adaptabilidad, las habilidades } \\
\text { sociales y gestión de conflictos } \\
\text { de los niños con TEA gracias al } \\
\text { perro de asistencia. }\end{array}$ \\
\hline $\begin{array}{l}\text { Wright, } \\
\text { Pasillo et al. } \\
\text { (2015, Reino } \\
\text { Unido) }\end{array}$ & $\begin{array}{l}\text { Conocer el impacto } \\
\text { de los perros en } \\
\text { niños con TEA, } \\
\text { concretamente los } \\
\text { niveles de estrés en } \\
\text { las familias. }\end{array}$ & $\begin{array}{l}\mathrm{N}=62 \text { padres } \\
\text { de niños con } \\
\text { TEA } \\
\mathrm{S}=50 \hat{0} 12 \text { } \\
\mathrm{E}=2-16 \text { años. }\end{array}$ & $\begin{array}{l}\text { Medición del estrés } \\
\text { parental por los cuidados } \\
\text { de un niño con TEA. }\end{array}$ & $\begin{array}{l}\text { Reducción del estrés del grupo } \\
\text { de padres que tienen perro de } \\
\text { asistencia para sus hijos con } \\
\text { TEA. }\end{array}$ \\
\hline $\begin{array}{l}\text { Wright, Hall et } \\
\text { al. (2015, } \\
\text { Reino Unido) }\end{array}$ & $\begin{array}{l}\text { Estudio del efecto de } \\
\text { la propiedad de un } \\
\text { perro de asistencia } \\
\text { sobre el } \\
\text { funcionamiento de la } \\
\text { familia y la ansiedad } \\
\text { del niño con TEA. }\end{array}$ & $\begin{array}{l}\mathrm{N}=70 \text { padres } \\
\text { de niños con } \\
\text { TEA. }\end{array}$ & $\begin{array}{l}\text { Evaluación del } \\
\text { funcionamiento familiar y } \\
\text { la ansiedad de los niños. }\end{array}$ & $\begin{array}{l}\text { Los perros de asistencia } \\
\text { consiguen reducir las dificultades } \\
\text { familiares, mejoran las fortalezas } \\
\text { y reducen la ansiedad en las } \\
\text { familias de niños con TEA. }\end{array}$ \\
\hline $\begin{array}{l}\text { Burgoyne et } \\
\text { al. (2014, } \\
\text { Irlanda) }\end{array}$ & $\begin{array}{l}\text { Examinar como los } \\
\text { perros de asistencia } \\
\text { afectan a la } \\
\text { seguridad infantil, la } \\
\text { recepción pública de } \\
\text { los niños con TEA y } \\
\text { los niveles de tensión } \\
\text { familiar. }\end{array}$ & $\begin{array}{l}\mathrm{N}=221 \text { padres } \\
\text { de niños con } \\
\text { TEA con perros } \\
\text { de asistencia } \\
\mathrm{E}=0-9 \text { años. }\end{array}$ & $\begin{array}{l}\text { Evaluación de los } \\
\text { resultados de la } \\
\text { adquisición de un perro de } \\
\text { asistencia en el hogar. }\end{array}$ & $\begin{array}{l}\text { Los perros mejoran la seguridad } \\
\text { de los niños con TEA, alivian los } \\
\text { niveles de tensión de las familias } \\
\text { y mejoran la recepción social de } \\
\text { los niños, no obstante, también } \\
\text { aumentan las responsabilidades } \\
\text { y obligaciones al tener un perro } \\
\text { de asistencia en el hogar. }\end{array}$ \\
\hline $\begin{array}{l}\text { Funahashi et } \\
\text { al. (2013, } \\
\text { New York) }\end{array}$ & $\begin{array}{l}\text { Examinar si las } \\
\text { intervenciones } \\
\text { asistidas con perros } \\
\text { pueden facilitar el } \\
\text { comportamiento } \\
\text { social positivo. }\end{array}$ & $\begin{array}{l}\mathrm{N}=2 \\
\text { Niño con TEA y } \\
\text { niño con } \\
\text { desarrollo } \\
\text { neurotípico } \\
\mathrm{E}=10 \text { años. }\end{array}$ & $\begin{array}{l}\text { Medición de las sonrisas } \\
\text { de los niños durante las } \\
\text { intervenciones con perros. }\end{array}$ & $\begin{array}{l}\text { Las sonrisas fueron aumentando } \\
\text { con el contacto con los perros, } \\
\text { dando lugar a realizaciones } \\
\text { exitosas de comunicación verbal } \\
\text { y no verbal y comportamientos } \\
\text { sociales positivos. Al principio de } \\
\text { las sesiones las sonrisas se } \\
\text { relacionaban con } \\
\text { comportamientos negativos, pero } \\
\text { con el paso de las sesiones, } \\
\text { cuando el niño sonreía, se } \\
\text { cumplía una relación social } \\
\text { positiva con el perro y las } \\
\text { personas de la sala, eliminando } \\
\text { las negativas. }\end{array}$ \\
\hline $\begin{array}{l}\text { Burrows et al. } \\
\text { (2008, } \\
\text { Canadá) }\end{array}$ & $\begin{array}{l}\text { Integración del perro } \\
\text { de asistencia en el } \\
\text { hogar de niños con } \\
\text { TEA. }\end{array}$ & $\begin{array}{l}\mathrm{N}=10 \text { niños con } \\
\mathrm{TEA} \\
\mathrm{S}=7 \hat{\delta} \text { 3 } \\
\mathrm{E}=4-14 \text { años. }\end{array}$ & $\begin{array}{l}\text { Seguimiento del perro de } \\
\text { asistencia en el } \\
\text { funcionamiento en el } \\
\text { hogar. }\end{array}$ & $\begin{array}{l}\text { Mejora en la compañía infantil, } \\
\text { aprendizaje de nuevas } \\
\text { habilidades, relajación, } \\
\text { recreación familiar, condición } \\
\text { social de la familia y la calidad } \\
\text { del sueño. }\end{array}$ \\
\hline
\end{tabular}

\section{Discusión}

Los artículos de investigación analizados puede dividirse en dos grupos, por un lado, encontramos artículos de investigación dirigidos a examinar los beneficios que aportan los perros de asistencia o servicio en el hogar de los niños con TEA a través de adultos significativos (Burrows et al. 2008; Burgoyne et al. 2014; Wright, Hall et al. 2015; Wright, Pasillo et al. 2015; Hall, Wright y Mills, 2016; Hall, Wright y Mills, 2017; Carlisle et al. 
2020; Hill, Ziviani y Driscoll, 2020) y, por otro lado, encontramos artículos donde el objeto de estudio es analizar las intervenciones asistidas por perros a niños con TEA (Funahashi et al. 2013; Guérin et al. 2018; Germone et al. 2019; Ávila et al. 2019; Barber y Proops, 2019; Uccheddu et al. 2019; Griffien et al. 2019; Protopopova et al. 2020; Silva et al. 2020: Morales et al. 2020; Hill, Ziviani, Discroll, Teoh, Chua y Cawdell, 2020).

Los artículos de investigación centrados en conocer los resultados de la relación o vínculo entre el perro de asistencia y los niños con TEA, tienen una muestra de participantes similares en número $(n=60-70)$, siendo todos padres de niños con TEA con edades comprendidas entre 2 y 18 años, los cuales tienen un perro de asistencia o servicio en el hogar (Burrows et al. 2008; Burgoyne et al. 2014; Wright, Hall et al. 2015; Wright, Pasillo et al. 2015; Hall, Wright y Mills, 2016; Hall, Wright y Mills, 2017; Carlisle et al. 2020), a diferencia del estudio de Hill, Zivani y Discroll (2020) en el que se entrevista a 6 terapeutas ocupacionales que realizan intervenciones asistidas con perros de terapias y se analizan los beneficios obtenidos de estas intervenciones. Mediante estos estudios se realiza una reflexión acerca de la integración del perro de asistencia en el hogar, los resultados obtenidos a través de la adquisición del perro de asistencia, especialmente los beneficios que causan a la familia y en el niño con TEA los vínculos que se crean entre el niño y el perro, el estrés parental y la carga de poseer un perro y la observación de algunos comportamientos de ansiedad de los perros (Burrows et al. 2008; Burgoyne et al. 2014; Wright, Hall et al. 2015; Wright, Pasillo et al. 2015; Hall, Wright y Mills, 2016; Hall, Wright y Mills, 2017; Carlisle et al. 2020).

Los resultados de estos análisis de investigación demuestran que las intervenciones asistidas con perros mejoran la comunicación verbal y no verbal de los niños con TEA (Funahashi et al. 2013; Germone et al. 2019; Ávila et al. 2019; Silva et al. 2020; Morales et al. 2020), lectura más fluida mejorando el ritmo de esta y su comportamiento, haciendo referencia a la postura (Barber y Prooprs, 2019), los perros crean en los niños con TEA motivación hacia la lectura (Uccheddu et al. 2019), aumentan los comportamientos sociales positivos y reducen los negativos (Griffien et al. 2019; Protopopova et al. 2020; Silva et al. 2020), mejoran la calidad de las interacciones sociales, aumentando su participación, los perros aumentan la motivación de los niños en las sesiones, reduciendo los esfuerzos de los profesionales en el desarrollo de su trabajo y, por lo tanto, tienen un comportamiento más adecuado hacia la tarea a desempeñar (Morales et al. 2020; Carlise et al. 2020; Hill, Ziviani, Discroll, Teoh, Chua y Cawdell, 2020; Hill, Ziviani y Discroll, 2020). De igual modo, los perros de servicio ofrecen aprendizajes de nuevas habilidades en los niños con TEA, aumentan la relajación y la adaptabilidad, intensifican la calidad del sueño, proliferan las interacciones sociales positivas y alivian los niveles de ansiedad de las familias, puesto que reducen las dificultades familiares ofreciendo más momentos de recreación familiar (Burrows et al. 2008; Burgoyne et al. 2014; Wright, Hall et al. 2015; Wright. Pasillo et al. 2015; Hall, Wright y Mills, 2016; Hall, Wright y Mills, 2017; Carlisle et al. 2020; Hill, Ziviani y Driscoll, 2020).

Sin embargo, estos estudios tienen varias limitaciones compartidas que habría que tener en cuenta para futuras investigaciones como son que los resultados obtenidos no son determinantes (Burgoyne et al. 2014; Wright y Pasillo, et al. 2015; Hall, Wright y Mills, 2016; Hall, Wright y Mills, 2017; Uccheddu et al. 2019; Carlisle et al. 2020; Hill, Ziviani y Driscoll, 2020), un reducido número de participantes en el estudio (Burrows et al. 2008; Funahashi et al. 2013; Ávila et al. 2019; Barber y Proops, 2019; Uccheddu et al. 2019; Griffien et al. 2019; Protopopova et al. 2020; Silva et al. 2020: Morales et al. 2020; Hill, Ziviani, Discroll, Teoh, Chua y Cawdell, 2020; Hill, Ziviani y Driscoll, 2020), un corto periodo de aplicación de la intervención sin seguimiento (Germone et al. 2019; Barber y Proops, 2019; Uccheddu et al. 2019; Silva et al. 2020), poca cantidad de estudios similares realizados para poder comparar los resultados alcanzados (Hall, Wright y Mills, 
2016; Wright, Pasillo et al. 2015) y ausencia de grupo de control en algunas investigaciones donde se comparen los resultados (Ávila et al. 2019).

En cuanto a los resultados obtenidos de los artículos de investigación sobre las intervenciones realizadas con perros a niños con TEA. Los participantes de los estudios son homogéneos en general, niños con TEA con edades comprendidas entre los 2 y los 12 años, predominando las intervenciones centradas en la etapa de Educación Primaria, exceptuando el artículo de Silva et al. (2020), el cual incluye en su estudio personas adultas con TEA, comprendiendo las edades de 9 a 38 años, en las cuales se ve una diferencia en los resultados, ya que, las personas adultas con TEA prefieren interactuar con los perros robóticos que con los perros de verdad. Así mismo, la cantidad de participantes $(\mathrm{N}=10)$ es similar en los estudios, y habiendo un predominio del género masculino entre los participantes, lo que concuerda con los datos sobre el TEA, que es cuatro veces más frecuente en el género masculino que en el femenino. Estudios como el de Germone et al. (2019) o Guérnin et al. (2018), superan la media de 13 participantes. En el caso de Funahashi et al. (2013), Guérin et al. (2018) y Barber y Proops (2019) añaden a sus estudios otros participantes con diferente diagnostico al TEA como es Síndrome de Down, trastorno por déficit de atención e hiperactividad, dislexia, ansiedad y fibrosis quística, encontrando que los estudiantes mejoraron sus habilidades en la lectura, tanto en la fluidez, como en el ritmo y el comportamiento de lectura respecto a la postura y confianza frente al perro y la mejora del bienestar animal en las intervenciones.

También se encuentran grandes diferencias sobre el procedimiento seguido en las intervenciones de los artículos de investigación, teniendo una duración y número de sesiones realizadas muy distintas entre todos ellos. De igual modo ocurre con el lugar de la intervención, ya que las sesiones son realizadas en lugares muy dispares, como en un hospital psiquiátrico (Germone et al. 2019), una Unidad asistencial de Hospital (Ávila et al. 2019), un aula de educación especial (Barber y Proops, 2019; Morales et al. 2020), una Universidad (Protopopova et al. 2020), casas de los participantes o en un centro de día (Burrows et al. 2008; Burgoyne et al. 2014; Wright, Hall et al. 2015; Wright, Pasillo et al. 2015; Hall, Wright y Mills, 2016; Hall, Wright y Mills, 2017; Silva, et al. 2020), involucrando profesionales sociales, de la salud y la educación. Las razas de los perros que realizan las intervenciones asistidas son muy variadas, no obstante, predominan las razas de Labrador o Golden Retriever, siendo todos certificados como perros de terapia (Funahashi et al. 2013; Guérin et al. 2018; Germone et al. 2019; Ávila et al. 2019; Barber y Proops, 2019; Uccheddu et al. 2019; Griffien et al. 2019; Protopopova et al. 2020; Silva et al. 2020; Hill, Ziviani, Discroll, Teoh, Chua y Cawdell, 2020) y perros de servicio o asistencia (Burrows et al. 2008; Burgoyne et al. 2014; Wright, Hall et al. 2015; Wright, Pasillo et al. 2015; Hall, Wright y Mills, 2016; Hall, Wright y Mills, 2017; Carlisle et al. 2020; Hill, Ziviani y Driscoll, 2020).

Las intervenciones realizadas son dispares en cuanto al objeto de estudio y tipología de la intervención, se encuentran diversos estudios que analizan la comunicación y el lenguaje, ya sea a través de la lectura (Barber y Proops, 2019; Uccheddu et al. 2019) o el análisis del lenguaje (Morales et al. 2020) o análisis del comportamiento, habilidades e interacciones sociales (Funahashi et al. 2013; Germone et al. 2019; Ávila et al. 2019;Griffiend et al. 2019; Silva et al. 2020; Hill, Ziviani, Discroll, Teoh, Chua y Cawdell, 2020). El estudio de Guérin et al. (2018) evalúa la herramienta OHAIRE la cual ha sido desarrollada para analizar el comportamiento de los niños durante las interactuaciones con interlocutores sociales o animales y, el artículo de investigación de Protopopova et al. (2020), estudia los niveles de cortisol en los niños con TEA al realizar tareas académicas con acceso contingente y no contingente con los perros de terapia para medir los niveles de ansiedad de los participantes a través de las muestras salivales extraídas al principio de la sesión y al final. 
Como se ha mencionado en el marco teórico de este trabajo, autores como la Fundación Affinity (2019) o Signes (2009) afirman que las IAA para los niños con TEA producen múltiples beneficios en el desarrollo y mejora de la calidad de vida de estos. Después de realizar este trabajo se ha podido constatar que estas afirmaciones son ciertas, reafirmándose los beneficios de estas intervenciones.

Las IAA, concretamente las intervenciones realizadas con perros de terapia mejoran las habilidades y relaciones sociales de los niños con TEA. Los perros ayudan a generar seguridad y confianza a los niños, de igual modo, crean entusiasmo y motivación en ellos, permitiéndoles desarrollar actividades y mantener la atención con más facilidad. Las intervenciones asistidas con perros reducen el estrés de los niños con TEA, los comportamientos negativos y la ansiedad, y, por consiguiente, producen comportamientos prosociales que les ayudan a establecer conductas sociales positivas. Además, los perros de asistencia que residen en los hogares con los niños con TEA no solo ayudan a dichos infantes, sino que también contribuyen en las relaciones familiares creando más espacios de recreación familiar, compensando de este modo el cuidado que pasa a tener la familia al adquirir un perro.

Por otro lado, las intervenciones asistidas con animales diferentes a los perros como pueden ser la equinoterapia o la delfinoterapia (Navarro, 2019), también ofrecen múltiples beneficios en el desarrollo de los niños con TEA, que se suman a los obtenidos de las interacciones con los perros. No obstante, en este trabajo se ha querido centrar el tema de estudio en las intervenciones con perros puesto que se considera que es el animal con más posibilidades y ventajas para en un futuro poder introducirlo en las aulas de los centros educativos de una manera factible y próxima en el tiempo.

Como se comenta en el artículo de Griffin et al. (2020) la sincronía que se crea entre los niños y los perros es un mecanismo subyacente de las IAA, el cual es precursor de establecer patrones de comportamiento social, los cuales pueden ser extendidos a las interacciones humanas que realizan los infantes con TEA. Del mismo modo, Funahashi et al. (2013) afirma que la relación entre los niños con TEA y los perros de terapia se crean bajo un "campo positivo de la emoción", donde el niño se siente seguro expresando sus propias emociones y siente sentimientos agradables. Así pues, los perros pueden llegar a ser precursores en las relaciones sociales de los niños con TEA, dado que pueden llegar a hacer que estos sientan las interacciones con el entorno y los estímulos que le rodean de una manera más positiva.

En cambio, hay que considerar que este tipo de intervenciones o terapias alternativas para los niños con TEA son relativamente nuevas $y$, como se ha podido observar, no se han encontrado intervenciones realizadas con perros de terapia en el ámbito educativo, más bien se relacionan con el ámbito social o de la salud. Por lo tanto, sería interesante poder realizar investigaciones futuras sobre la viabilidad y limitaciones que puede llegar a tener la implantación de la intervención asistida con perros en las aulas de pedagogía terapéutica de los centros educativos, dado que como afirma Hill, Zivani, Discroll, Teoh, Chua y Cawdell (2020), el contar con un perro de terapia no solo facilita el desarrollo de las sesiones o tareas al alumnado con TEA, sino que el esfuerzo de las personas responsables en llevar a cabo las sesiones se ve reducido, debido a que el perro ayuda al profesional a que los niños se concentren, estén motivados y desarrollen las sesiones con más autonomía.

Otro aspecto para tener en cuenta es que en ningún artículo de investigación se ha encontrado información relativa al bienestar del animal, a excepción del artículo de Hall, Wright y Mills (2017), en el cual sí que se describen una serie de factores negativos para la calidad de vida de los perros. Por tanto, otra posible investigación futura sería analizar 
el comportamiento e indicaciones de estrés del perro para asegurar que el animal desempeñe su trabajo en las condiciones óptimas y que no interfieran o afecten las sesiones a su bienestar e integridad.

Finalmente, este trabajo abre nuevas cuestiones para futuras investigaciones como son implementar las intervenciones asistidas con perros en las aulas de los centros educativos o analizar qué factores pueden ser de riesgo en las sesiones para asegurar que el perro de terapia tenga una mejor calidad de vida.

En la actualidad, las IAA se ven como actividades o terapias alternativas no farmacológicas en la educación de las personas con TEA, por tanto, sería importante investigar en profundidad este tema para poder implantar estas intervenciones en las escuelas que tantos beneficios aportan a los niños con TEA.

Además, falta investigación y formación para los docentes en este ámbito cómo adiestrar al perro de terapia, cómo introducir al animal en las sesiones, qué cuidados debe tener para conservar su salud e integridad, cómo saber cuándo el can está sufriendo, qué actividades realizar y qué objetivos plantear según qué área (cognitiva, física o relacional) se quiera trabajar. Debido a lo cual, este estudio sirve como trabajo previo el cual plantea nuevos caminos para poder seguir analizando la implementación de las intervenciones asistidas con perros en el aula para alumnado con TEA, favoreciendo su desarrollo e inclusión.

\section{Referencias}

American Psychiatric Association - APA. (2013). Manual diagnóstico y estadístico de los trastornos mentales DSM-5 (5a. ed.). Madrid: Editorial Médica Panamericana.

Ávila, A., Alonso, M., De Rosende, I., Vizcaíno, M., Larrañeta, L., y Torres, G. (2019). Improving social participation of children with autism spectrum disorder: Pilot testing of an early animal-assisted intervention in Spain. Health and social care in the community, 28(4), 1220-1229. https ://doi.org/10.1111/hsc.12955

Barber, O., y Proops, L. (2019). Low-ability secondary school students show emotional, motivational, and performance benefits when reading to a dog versus a teacher. Anthrozoös, 32(4), 503518. DOI: 10.1080 / 08927936.2019 .1621522

Bonilla, M., y Chaskel, R. (2016). Trastorno del espectro autista. Programa de Educación continua en Pediatría. Sociedad Colombiana de Pediatría, 15(1), 19-29. https://scp.com.co/wpcontent/uploads/2016/04/2.-Trastorno-espectro.pdf

Burgoyne, L., Dowling, L., Fitzgerald, A., Connolly, M., Browne, J. y Perry, I. (2014). Parents' perspectives on the value of assistance dogs for children with autism spectrum disorder: a cross-sectional study. BMJ open, 4(6), 1-10. doi:10.1136/bmjopen-2014-004786

Burrows, K. E., Adams, C. L., y Spiers, J. (2008). Sentinels of safety: Service dogs ensure safety and enhance freedom and well-being for families with autistic children. Qualitative health research, 18(12), 1642-1649. DOI: $10.1177 / 1049732308327088$

Carlisle, G. K., Johnson, R. A., Wang, Z., Brosi, T. C., Rife, E. M. y Hutchison, A. (2020). Exploring Human-Companion Animal Interaction in Families of Children with Autism. Journal of autism and developmental disorders, 50(8), 2793-2805. https://doi.org/10.1007/s10803-020-04390-x
Chaste, P., \& Leboyer, M. (2012). Autism risk factors: genes, environment, and gene-environment interactions. Dialogues in clinical neuroscience, $14(3), \quad 281$. DOI:10.31887/DCNS.2012.14.3/pchaste

Fombonne, E. (2003). The prevalence of autism. Journal of the American Medical Association, 289(1), 87-89. https://doi.org/10.1001 / jama.289.1.87

Fortea Sevilla, M., Escandell Bermúdez, M., \& Castro Sánchez, J. J. (2013). Detección temprana del autismo: profesionales implicados. Revista Española de Salud Pública, 87(2), 191-199. https://dx.doi.org/10.4321/S1135-57272013000200008

Funahashi, A., Gruebler, A., Aoki, T., Kadone, H. y Suzuki, K. (2013). Brief report: the smiles of a child with autism spectrum disorder during an animal-assisted activity may facilitate social positive behaviors - quantitative analysis with smile-detecting interface. Journal of autism and developmental disorders, 44(3), 685-693. DOI 10.1007/s10803-013-1898-4

Fundación Affinity. (2019). Terapias Asistidas con Animales y personas con autismo. https://www.fundacionaffinity.org/blog/terapias-asistidas-con-animales-y-personas-conautismo-2019

Germone, M. M., Gabriels, R. L., Guérin, N. A., Pan, Z., Banks, T. y O'Haire, M. E. (2019). Animal-assisted activity improves social behaviors in sychiatrically hospitalized youth with autism. Autism, 23(7), 1740-1751. DOI: 10.1177/1362361319827411

Griffin, R. E., van der Steen, S., Verheggen, T., Enders, M. J., y Cox, R. (2020). Changes in behavioural synchrony during dog-assisted therapy for children with autism spectrum disorder and children with Down syndrome. Journal of Applied Research in Intellectual Disabilities, 33(3), 398-408. DOI: 10.1111/jar.12682 
Guérin, N. A., Gabriels, R. L., Germone, M. M., Schuck, S. E., Traynor, A., Thomas, K. M., Mackzine, S., Slaughter, V. y O'Haire, M. E. (2018). Reliability and validity assessment of the observation of human-animal interaction for research (OHAIRE) behavior coding tool. Frontiers in veterinary science, 5, 1-15. doi: 10.3389/fvets.2018.00268

Hall, S. S., Wright, H. F. y Mills, D. S. (2016). What factors are associated with positive effects of dog ownership in families with children with autism spectrum disorder? The development of the Lincoln autism pet dog impact scale. PLoS One, 11(2), 1-19. doi: 10.1371/journal.pone. 0149736

Hall, S. S., Wright, H. F. y Mills, D. S. (2017). Parent perceptions of the quality of life of pet dogs living with neuro-typically developing and neuro-atypically developing children: An exploratory study. PloS one, 12(9), 1-22. https://doi.org/10.1371/journal.pone.0185300

Hill, J., Ziviani, J. y Driscoll, C. (2020). "The connection just happens": Therapists' perspectives of canine-assisted occupational therapy for children on the autism spectrum. Australian Occupational Therapy, 67(6), 550- 562. https://doi.org/10.1111/1440-1630.12680

Hill, J., Ziviani, J., Driscoll, C., Teoh, A. L., Chua, J. M., y Cawdell, J. (2020). Canine assisted occupational therapy for children on the autism spectrum: A pilot randomised control trial. Journal of autism and developmental disorders, 50(11), 4106-4120. https://doi.org/10.1007/s10803-020-04483-7

Morales-Moreno, I., Cerezo-Chuecos, F., Balanza-Galindo, S. Gómez-Díaz, M. y Echevarría-Pérez, P. (2020). Implementation of Assisted Therapy With Dogs in the Therapeutic Approach to People With Autistic Spectrum Disorder. Holistic Nursing Practice, 34(5), 282-290. DOI: $10.1097 / \mathrm{HNP} .0000000000000403$

Navarro, P. N. (2019). Intervención asistida con animales y su beneficio en el tratamiento del trastorno del espectro autista en niños y adolescentes (Tesis de licenciatura).

Protopopova, A., Matter, A., Harris, B., Wiskow, K. y Donaldson, J. (2020). Comparison of contingent and noncontingent access to therapy dogs during academic tasks in children with autism spectrum disorder. Journal of applied behavior analysis, 53(2), 811834.

Ristol, F. y Domènec, E. (2013). Terapia Asistida con Animales. Método CTAC. Técnicas y Ejercicios para Intervenciones Asistidas con Perros. Smiles CTAC.

Sanz-Cervera, P., \& Tárraga-Mínguez, R. (2018). ¿Qué estrategias de intervención funcionan en la educación de los niños con trastorno del espectro autista? Revisión de evidencias en la literatura científica. ReiDoCrea, 7. DOI:10.30827/Digibug.54134

Silva, K., Lima, M., Fafiães, C., Sinval, J. y de Sousa, L. (2020). Preliminary test of the potential of contact with dogs to elicit spontaneous imitation in children and adults with severe autism spectrum disorder. The American Journal of Occupational Therapy, 74(1), 7401205070p1-7401205070p8.

Uccheddu, S., Albertini, M., Pierantoni, L., Fantino, S. y Pirrone, F. (2019). The impacts of a Reading-to-Dog Programme on attending and reading of nine children with Autism Spectrum Disorders. Animals, 9(8), 1-12. doi:10.3390/ani9080491

Urrútia, G y Bonfill, X. (2010) Declaración PRISMA: una propuesta para mejorar la publicación de revisiones sistemáticas y metaanálisis. Medicina clínica, 135(11), 507-511. DOl: 10.1016/j.medcli.2010.01.015

Wright, H., Hall, S., Hames, A., Hardiman, J., Mills, R., PAWS Project Team, y Mills, D. (2015). Pet dogs improve family functioning and reduce anxiety in children with autism spectrum disorder. Anthrozoös, 28(4), 611-624. https://doi.org/10.1080/08927936.2015.1070003

Wright, H., Pasillo, S., Hames, A., Hardiman, J., Molinos, R., Molinos, D. (2015). Acquiring a pet dog significantly reduces stress of primary carers for children with Autism Spectrum Disorder: A prospective case control study. Journal of autism and developmental disorders, 45(8), 2531-2540. DOI 10.1007/s10803015-2418-5 